$\Gamma^{\prime}(x) / \Gamma(x)=\Psi(x)$ give a very easy way to compute as many terms as may be desired in the series which occurs in the asymptotic form of the gamma function.

INDIANA UNIVERSITY, March, 1913.

\title{
AN ERRONEOUS APPLICATION OF BAYES' THEOREM TO THE SET OF REAL NUMBERS.
}

BY DR. EDWARD L. DODD.

(Read before the American Mathematical Society, January 1, 1913.)

BAYEs' theorem on the probability of causes is frequently introduced with an urn problem.* Here only a finite number of objects come into consideration. For example: The urn $U_{1}$ contained 3 white balls and 1 black ball; the urn $U_{2}$ contained 2 white balls and 2 black balls. A man, blindfolded, drew a white ball. What is the probability that this white ball came out of $U_{1}$,- assuming that each urn was equally accessible? After a consideration of the general problem of this nature, the following theorem, known as Bayes' theorem, is announced:

Let $\omega_{i}$ be the probability a priori that a certain urn, or "cause," or set of conditions $U_{i}$ will come into play. The "causes" are to be mutually exclusive; and $i=1,2, \cdots, s$. Let $p_{i}$ be the probability that $U_{i}$, when brought into play, will yield a certain event. Then, after this event has happened, the probability a posteriori $P_{i}$ that the event had its origin in $U_{i}$ is

$$
P_{i}=\frac{\omega_{i} p_{i}}{\omega_{1} p_{1}+\omega_{2} p_{2}+\cdots+\omega_{s} p_{s}} .
$$

In the preceding example, it is assumed that $\omega_{1}=\omega_{2}=\frac{1}{2}$. Hence, with $p_{1}=\frac{3}{4}, p_{2}=\frac{2}{4}$, it follows that $P_{1}=\frac{3}{5}$, - a result which on inspection seems plausible; since $\frac{3}{5}$ of all the white balls were in the first urn, $U_{1}$. This urn example illustrates, indeed, the following important corollary of Bayes' theorem: If each of a finite number $s$ of mutually exclusive causes

* E. g., Poincaré, Calcul des Probabilités (1912), p. 153. 
is equally likely a priori to come into play, then the probability a posteriori that a given event had its origin in a specified cause is proportional to the probability that the specified cause would produce that event.

In applying Bayes' theorem in the theory of errors of measurements, Poincaré* regards a possible value for the unknown as an ideal " cause." He lets $z$ be any real number; and writes

$$
\omega_{i}=\psi(z) d z
$$

as the probability a priori that the true value of the unknown will lie between $z$ and $z+d z$. With the true value in this interval, the probability that the first measurement will then lie between $x_{1}$ and $x_{1}+d x_{1}$ is written as

$$
d x_{1} \varphi\left(x_{1}, z\right) \text {. }
$$

If, now, the $n$ measurements have been found to lie between $x_{1}$ and $x_{1}+d x_{1}, \cdots, x_{n}$ and $x_{n}+d x_{n}$, respectively,-more briefly: to be $x_{1}, x_{2}, \cdots, x_{n}$, respectively, - then the probability a posteriori that the true value lies between $z$ and $z+d z$ is found to be

$$
\frac{d z \psi(z) \varphi\left(x_{1}, z\right) \varphi\left(x_{2}, z\right) \cdots \varphi\left(x_{n}, z\right)}{\int_{-\infty}^{+\infty} d z \psi(z) \varphi\left(x_{1}, z\right) \varphi\left(x_{2}, z\right) \cdots \varphi\left(x_{n}, z\right)} .
$$

Poincaré then points out how Gauss by taking $\psi=1$, by assuming that $\varphi$ is a function of $z-x_{i}$, and by assuming that the arithmetic mean is the most probable value of the unknown, reaches the conclusion that

$$
\varphi(y)=\sqrt{\frac{h}{\pi}} e^{-h y^{2}},
$$

where $y$ is the error, $z-x$.

Poincaré then† mentions Bertrand's objections to the above assumptions, saying in part: "De plus, on a fait $\psi(z)=1$, et l'on ne peut l'affirmer a priori."

We may add that the assumption that $\psi(z)$ is any constant for all real values of $z$ is not only unwarranted, but it is analytically impossible. For if $z$ is to have the range from $-\infty$

* Loc. cit., p. 169.

†P. 173. 
to $+\infty$, the probability function $\psi(z)$ must satisfy the condition

$$
\int_{-\infty}^{+\infty} \psi(z) d z=1
$$

since it is certain that the number expressing the measure of the quantity lies between $-\infty$ and $+\infty$. This condition cannot be satisfied if $\psi$ is a constant.

This equation, $\psi=$ constant, seems to be the symbolic equivalent of the statement that, before measurements are made, all real numbers have equal probability a priori of being the true value,-if, indeed, we can attach any useful meaning to this statement.

The difficulty of so doing appears to have its origin in the fact that the set of real numbers is unbounded; rather than that the set contains an infinity of individuals. To illustrate: Suppose that a rod of unknown length lies in a narrow box with interior length of 20 inches. We may, then, with some propriety, set $\psi(z)=1 / 20$; and let $z$ range from 0 to 20 . By so doing we would at least satisfy the requirement,

$$
\int \psi(z) d z=1,
$$

taken over all the values of $z$ possible in the given case. Furthermore, this probability may be interpreted as an ideal frequency. For instance, the probability that the length lies between 11.2 inches and 11.3 inches would be 1/200. This would signify that in 100,000 such boxes put up with rods taken by chance,* we should expect to find about 500 boxes containing rods with length between 11.2 inches and 11.3 inches.

The extension of these conceptions to the range from $-\infty$ to $+\infty$ does not seem to be immediate. Is the probability that the true value lies between 50 inches and 51 inches the same as the probability that the true value lies between one light-year and one light-year plus one inch? If so what is the probability in each case? That the generalization in question should present some difficulty is not surprising, in view of the fundamental difference between a proper integral and an improper integral.

This leaves one more hiatus in the argument that attempts

* It is not asserted here that under these circumstances we must take $\psi(z)=1 / 20$. 
to deduce the probability law (1) from the so-called principle of the arithmetic mean,- - as the "most probable value." The argument referred to is that which first sets up

$$
F(z)=\varphi\left(z-x_{1}\right) \varphi\left(z-x_{2}\right) \cdots \varphi\left(z-x_{n}\right) d y_{1} d y_{2} \cdots d y_{n}
$$

as the probability that, with $z$ as the true value, the measurements $x_{1}, x_{2}, \cdots, x_{n}$, will be made; then attempts to regard this same expression as also proportional-or even equal-to the probability that $z$ is the true value, the measurements $x_{1}$, $x_{2}, \cdots, x_{n}$, having been made; and then sets $d F / d z=0$.

UNIVERSity of TeXas, December, 1912.

\section{SHORTER NOTICES.}

Die partiellen Differential-Gleichungen der mathematischen Physik. Nach Riemann's Vorlesungen in fünfter Auflage bearbeitet von HeInRICH Weber. Zweiter Band. Braunschweig, Vieweg und Sohn, 1912. xiv+575 pp. Unbound 15 marks, bound 16.80 marks.

The first volume of the fifth edition of this classic work was reviewed in this Bulletin, volume 18 , page 87 , and the fourth edition in volume 8 , page 81 . Little need be added to these. The most noteworthy addition to the present volume is the entire section 18, devoted to relativity. This section contains thirty pages. The introduction points out the nature of time and that relativity is not really concerned with time but with the measure of time, or rather with the connection between time and space quantity. The succeeding sections are sufficiently described by their titles: time and space in the stationary and the moving world; normal form of the transformation of axes; constant velocity of light; significance of the Lorentz transformation; the fundamental electromagnetic equations for bodies at rest; the fundamental electromagnetic equations for moving bodies; invariancy of the equations; explicit form of the equations; transformation of the force and the displacement; the Michelson-Morley experiment; application of the relativity theory to the Michelson- 